\title{
Endovascular treatment of distal posterior inferior cerebellar artery aneurysms
}

\author{
Jun Tang, $M D$, Linjie Wei, $M D$, Lin Li, MD, Yin Niu, MD, Qianwei Chen, MD, Hua Feng, $M D, P h D$, Gang Zhu, MD, PhD, \\ Zhi Chen, $M D$, $P h D$.
}

\begin{abstract}

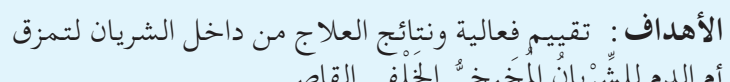

الطريقة : قمنا بإِجراء دراسة استرجاعية ومراجعة خبراتنا ونتائج

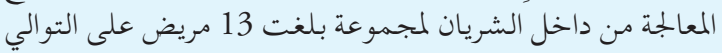

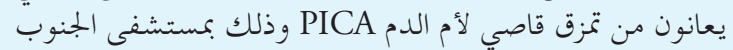

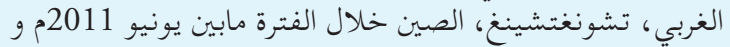

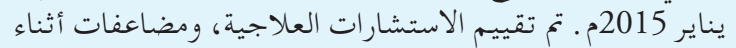

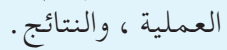

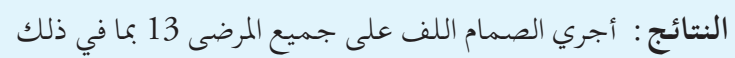

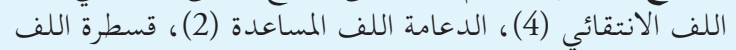

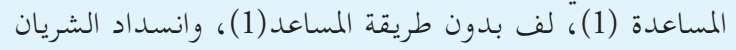

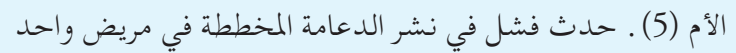

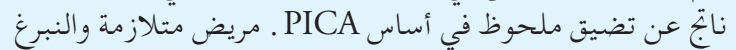

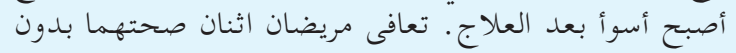

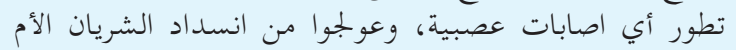

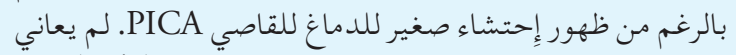

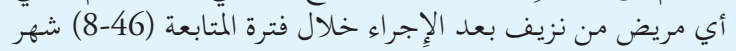
متوسط 26.8 شهر مرن.

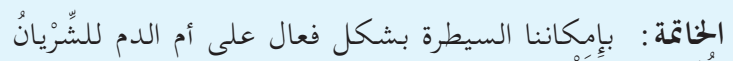

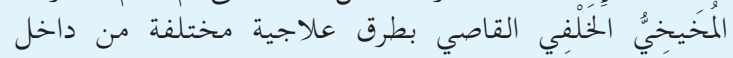

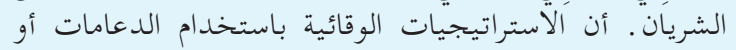

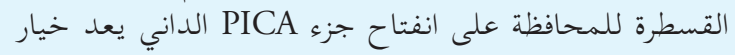

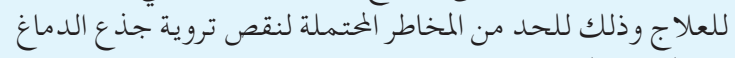

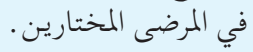

Objective: To assess the feasibility and results of endovascular treatment for ruptured distal posterior inferior cerebellar artery (PICA) aneurysms.

Methods: We retrospectively reviewed our experience and results with endovascular treatments for a series of 13 consecutive patients with ruptured distal PICA aneurysms at the Southwest Hospital, Chongqing,
China, treated between June 2011 and January 2015. Therapeutic considerations, intraoperative complications, and results were evaluated.

Results: Endovascular embolization was performed in all 13 patients including selective coiling (4), stentassisted coiling (2), microcatheter-assisted coiling (1), coiling without adjunctive techniques (1), and parent artery occlusion (5). Planed stent deployment failure occurred in one patients resulting from marked stenosis at the origin of the PICA; the patient's existing partial Wallenberg's syndrome became worse after treatment. Another 2 patients treated with parent artery occlusion recovered well without developing obvious neurological deficits, although a small cerebellar infarction of the distal PICA was observed. No patient experienced post procedural hemorrhage during the follow-up period (8-46 months; mean, 26.8 months).

Conclusion: Distal PICA aneurysms can be managed effectively with various endovascular methods. Protective strategies using a stent or microcatheter for preserving the patency of the proximal PICA segment are a viable treatment option in reducing the potential risk of brain stem ischemia in selected patients.

Neurosciences 2016; Vol. 21 (3): 236-240 doi: 10.17712/nsj.2016.3.20160076

From the Department of Neurosurgery (Tang, Li, Niu, Chen Q, Feng, Zhu, Chen Z), Southwest Hospital, Third Military Medical University, Chongqing, and the Department of Neurosurgery (Wei), PLA 115th Hospital, Lin Zhi, Tibet, China.

Received 7th February 2015. Accepted 9th March 2016.

Address correspondence and reprint request to: Dr. Zhi Chen, Professor of Neurosurgery, Department of Neurosurgery, Southwest Hospital, Third Military Medical University, No. 30 Gaotanyan Street, Chongqing 400038, China. E-mail: zhichent@tmmu.edu.cn

Cerebral aneurysms of the posterior inferior cerebellar artery (PICA) are rare, representing approximately $0.5-3 \%$ of all intracranial aneurysms. ${ }^{1-3}$ These aneurysms usually arise at the vertebral artery 
(VA)-PICA junction, while distal PICA aneurysms only account for less than $30 \%$ of all PICA aneurysms. ${ }^{3}$ Traditionally, most cases have been treated surgically, including clipping, wrapping, or trapping with or without vascular anastomosis. ${ }^{2-5}$ However, surgical treatment for those aneurysms can carry a significant risk of morbidity due to the close relationship of PICA aneurysms with the brain stem and lower cranial nerves. Although endovascular embolization as an alternative treatment has been recommended as a primary option in treating PICA aneurysms by some authors recently, ${ }^{1,4}$ there is still controversy regarding therapeutic strategies and complications. In the present study, we report our experience with, and associated complications of endovascular treatment of distal PICA aneurysms.

Methods. Thirteen consecutive patients with distal PICA aneurysms treated with endovascular embolization between June 2011 and January 2015 were included in the present study. This study was approved by the Ethics Committee of the Southwest Hospital, and was carried out under the tenets of the Helsinki declaration. As shown in Table 1, our patients consisted of 5 females and 8 males with a mean age of 57 years (range, 39-73 years). All patients presented with acute subarachnoid hemorrhage and intraventricular hemorrhage. According to the criteria described by Lister et $\mathrm{al},{ }^{6}$ the locations of the distal PICA artery aneurysms in our group were divided into 5 segments: anterior-medullary (AM) in 3 cases, lateral-medullary (LM) in 3 cases, tonsillomedullary (TM) in one case, telovelotonsillar (TT) in 4 cases, and cortical in 2 cases.

Endovascular treatment was performed under general anesthesia, and systemic heparinization was administered during the procedure. All of the procedures were performed via the transfemoral route. Depending on the vascular anatomy and location of the aneurysms at the distal PICA, various therapeutic strategies were chosen including selective aneurismal coiling, parent artery occlusion, microcatheter-assisted coiling, and stent-assisted coiling. For saccular-shaped aneurysms with a favorable neck, selective aneurysmal coiling was attempted. Microcatheter-assisted coiling or stentassisted coiling was used for wide-necked aneurysms with preservation of the PICA. For fusiform aneurysms at the more proximal PICA segments (anteriormedullary and lateral-medullary), stent-assisted coiling was considered firstly. The parent artery, together with the aneurysm occlusion (endovascular trapping) using coils or glue, was carried out in fusiform aneurysms at the more distal PICA segments (telovelotonsillary and cortical segments).

The standard methods of aneurysm coiling, stentassisted coiling, or microcatheter-assisted coiling were used as previously described. ${ }^{7}$ A loading dose of aspirin $(300 \mathrm{mg})$ and clopidogrel $(225 \mathrm{mg})$ was administered orally 2 hours before stenting. The Enterprise selfexpanding stent (Codman, Miami Lakes, FL, USA)

Table 1 - Clinical characteristics of patients with distal aneurysms of the posterior inferior cerebellar artery.

\begin{tabular}{|c|c|c|c|c|c|c|c|}
\hline Case & Age/gender & $\begin{array}{l}\mathrm{H}-\mathrm{H} \\
\text { Grade }\end{array}$ & Site of the aneurysm & Shape & Treatment & $\begin{array}{l}\text { Immediate } \\
\text { result }\end{array}$ & Complications \\
\hline 1. & $73 / \mathrm{M}$ & I & Lateral-medullary & Saccular & Selective coiling & Complete & None \\
\hline 2. & $39 / \mathrm{M}$ & III & Anterior-medullary & Fusiform & Trapping & Complete & Lateral medulla oblongata infarct \\
\hline 3. & $65 / F$ & II & Tonsillomedullary & Saccular & Selective coiling & Complete & None \\
\hline 4. & $57 / \mathrm{M}$ & II & Telovelotonsillar & Fusiform & Trapping & Complete & Unilateral cerebellar infarcts \\
\hline 5. & $56 / \mathrm{F}$ & III & Anterior-medullary & Saccular & Stenting/coiling & Complete & Intraoperative rupture \\
\hline 6. & $68 / \mathrm{M}$ & IV & Cortical & Fusiform & Trapping & Complete & None \\
\hline 7. & $58 / \mathrm{M}$ & I & Anterior-medullary & Saccular & Selective coiling & Complete & None \\
\hline 8. & $61 / \mathrm{M}$ & III & Telovelotonsillar & Saccular & $\begin{array}{l}\text { Microcatheter } \\
\text { protection/coiling }\end{array}$ & Complete & None \\
\hline 9. & $42 / \mathrm{M}$ & II & Lateral-medullary & Fusiform & Trapping & Complete & None \\
\hline 10. & $55 / \mathrm{M}$ & III & Lateral-medullary & Fusiform & Stenting/coiling & Near complete & None \\
\hline 11. & $57 / \mathrm{F}$ & II & Cortical & Fusiform & Trapping & Complete & Unilateral cerebellar infarcts \\
\hline 12. & $61 / \mathrm{F}$ & II & Telovelotonsillar & Fusiform & Trapping & Complete & None \\
\hline 13. & $50 / \mathrm{F}$ & II & Telovelotonsillar & Saccular & Selective coiling & Complete & None \\
\hline
\end{tabular}


was used for stent-assisted coiling. Detachable coils and liquid embolic material (Glubran or Onyx-18) were used for embolization. After endovascular treatment, patients with stent placement were given dual antiplatelet therapy (75 mg clopidogrel, and 100 $\mathrm{mg}$ aspirin once a day) orally for one month, followed by aspirin alone for at least 3 months. Heparinization was continued for 48 hours in patients who underwent parent artery occlusion. Follow-up evaluation was based on physical examination and imaging studies, including conventional angiography or magnetic resonance angiography.

Results. Endovascular embolization was performed in all patients. Of 6 patients with saccular aneurysms, selective embolization was achieved without adjunctive techniques in 4 patients (Figure 1), while microcatheterassisted coiling or stent-assisted coiling was required in 2 patients. For 2 patients with fusiform aneurysms at the anterior-medullary or lateral-medullary segment of the PICA, stent-assisted coiling was preferentially considered. In the first patient, navigation of the microcatheter for stent delivery failed either together with the microcatheter for coiling or after the coil embolization due to marked stenosis at the origin of the PICA (Figure 2). Therefore, aneurysm occlusion together with PICA was carried out. In the subsequent patient, we inserted a 0.014-inch exchange microguidewire into the distal PICA before coiling of the aneurysm. After the fusiform aneurysm and the involved segment of PICA was partially coiled, the microcatheter was withdrawn, and the stent delivery and deployment were carried out along the inserted exchange microguidewire to maintain the patency of the PICA (Figure 3). The parent vessel occlusions were performed in the remaining 5 patients with fusiform aneurysms at the more distal PICA segments (Figure 4).

There was complete obliteration in 12 patients, and nearly complete occlusion in one patient. An intraoperative rupture of the aneurysm occurred in one patient with a saccular aneurysm treated with stentassisted coiling. The coil packing procedure was then continued until complete occlusion was achieved, and the patient recovered well. Two patients underwent external ventricular drainage after endovascular

Disclosure. Authors declare no conflicting interests, and the study was not supported/funded by any drug company. This study was supported by the National Key Technology R \& D Program of the Ministry of Science and Technology of China (2011BAI08B06).
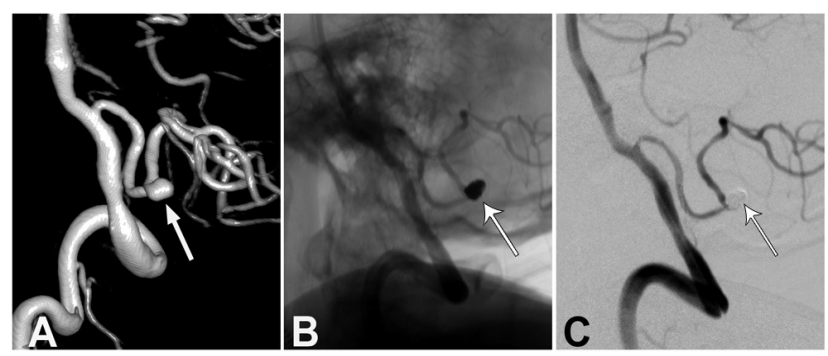

Figure 1 - Endovascular treatment for a patient with a saccular-shaped aneurysm at the distal posterior inferior cerebellar artery (PICA) A) Three-dimensional angiogram of the right vertebral artery angiogram saccular-shaped aneurysm (arrow) at the distal PICA. B \& C) Angiogram obtained immediately after embolization showing total occlusion of the aneurysm (arrow). B) unsubtracted angiogram and C) subtracted angiogram

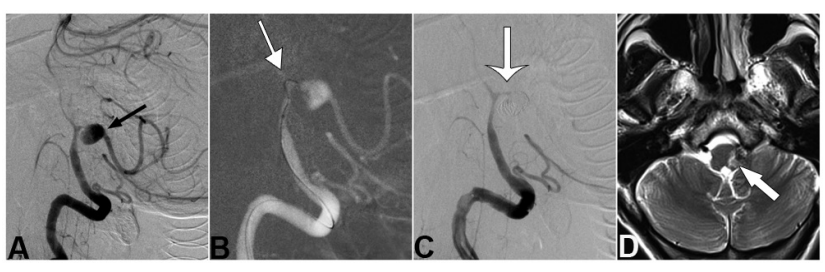

Figure 2 - Endovascular treatment for a patient with a fusiform aneurysm at the distal posterior inferior cerebellar artery (PICA). A) Left vertebral artery angiogram showing a fusiform aneurysm at the distal PICA associated with marked narrowing of the proximal PICA (arrow). B) Road mapping image showing difficulties in navigating the microguidewire and microcatheter through the narrowing segment of the proximal PICA (arrow). C) Angiogram obtained immediately after embolization showing complete occlusion of the aneurysm as well as the parent artery (arrow). D) Postoperative T2-weighted MRI demonstrating lateral-medullary infarction (arrow).

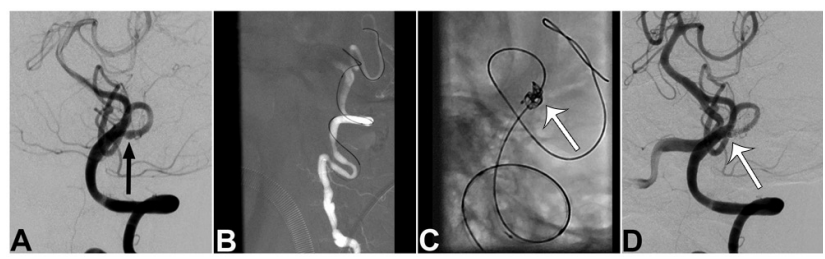

Figure 3 - Endovascular treatment for a patient with a fusiform aneurysm at the distal posterior inferior cerebellar artery (PICA). A) Left vertebral artery angiogram showing a fusiform aneurysm (arrow) at the distal PICA. B) Road mapping image showing an exchange microguidewire navigated to the distal PICA through the tortuous left vertebral artery before coiling of the aneurysm. C) Fluoroscopic image showing the coil delivery in the fusiform dilation before the subsequent stent deployment. D) Angiogram obtained immediately after embolization showing the disappearance of the fusiform dilation and patency of the PICA (arrow). 


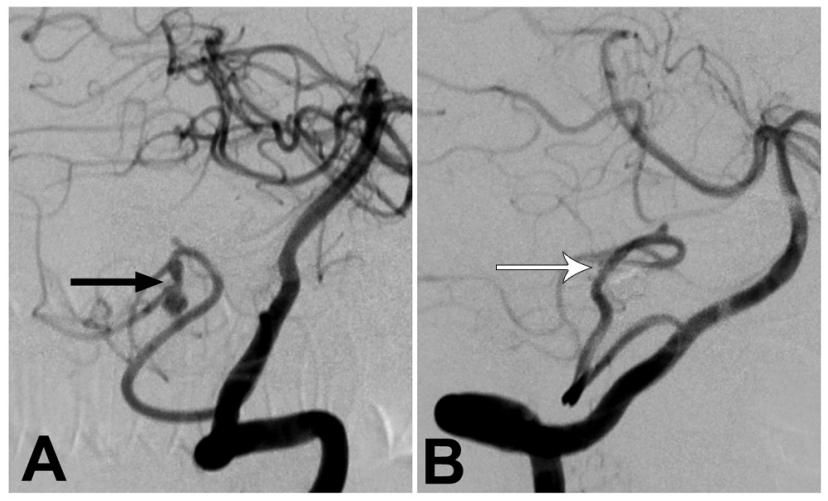

Figure 4 - Endovascular trapping for a patient with a fusiform aneurysm at the distal PICA A) Left vertebral artery angiogram showing a fusiform aneurysm (arrow) at the cortical segment of the posterior inferior cerebellar artery. B) Angiogram obtained immediately after embolization showing total occlusion of the aneurysm together with the involved branch.

treatment. No patient experienced post procedural hemorrhage in our group. Postoperative MR imaging revealed ipsilateral PICA territory infarction in 3 of 6 patients treated by parent artery occlusion. Infarction of the lateral medulla oblongata was observed in the first patient treated by parent artery occlusion with coils at the anterior-medullary segment of the PICA (Case 2, Figure 2), whose existing partial Wallenberg's syndrome worsened after treatment. He recovered gradually with mild dysphagia and ataxia. In the remaining 2 patients, although a small cerebellar infarction of the distal PICA was observed, the patients recovered well without developing obvious neurological deficits. Imaging, including CT angiogram, MR angiogram, or digital subtraction angiogram, and clinical follow-up (range 8-46 months; mean, 26.8 months) were available for all patients. No patient experienced post procedural hemorrhage or delayed cerebral infarction. Stable occlusion was found with no signs of recanalization in all patients.

Discussion. The reported incidence of distal PICA aneurysms varies from $0.38-1.7 \%$ of all intracranial aneurysms. ${ }^{3}$ The pathophysiology leading to the formation of these artery aneurysms is not well known. Some of these aneurysms were found to be associated with a cerebellar arteriovenous malformation (AVM), in which the increased flow dynamical stress resulting from the AVM may have contributed to the formation and rupture of the aneurysms. Lewis et $\mathrm{al}^{2}$ reported 20 patients with 22 distal PICA aneurysms, and 6 of the 20 patients $(30 \%)$ had associated cerebellar AVMs. In addition, several traumatic PICA aneurysms have been illustrated in sporadic single-case reports. ${ }^{8}$ Compared with other cerebral aneurysms at the arterial branch points around the circle of Willis, distal aneurysms are more likely to be fusiform. ${ }^{9}$ According to previous reports, ${ }^{2,10,11}$ most fusiform aneurysms, and some wide-necked aneurysms of the distal PICA represent arterial dissection with pseudoaneurysms, although typical angiographic findings of dissection may not be observable in small distal PICA. In our series, PICA aneurysms associated with AVM were not included. Of 13 distal PICA aneurysms, 6 (46\%) were saccular, and 7 (54\%) were fusiform. Consistent with previous reports, ${ }^{2-4}$ the most common location of the aneurysms in our series was the TT segment of the PICA (4/13), possibly because of the hemodynamic stress at its curvature.

Endovascular treatment of a distal PICA aneurysm could technically be performed either by selective embolization with parent artery preservation, or by parent artery occlusion. These aneurysms usually have a fusiform shape or wide neck, making selective embolization with parent artery preservation difficult, or impossible in a substantial percentage of cases. Therefore, endovascular parent artery occlusion is generally considered an appropriate and reliable alternative treatment for ruptured distal PICA aneurysms, especially for fusiform or dissecting aneurysms. ${ }^{10,12}$ Isokangas et $\mathrm{al}^{12}$ reported 12 patients with peripheral PICA aneurysms, and 6 patients were treated with parent artery occlusion, of which only 2 patients had transient postoperative neurological deficits. In a recent series of 10 patients with ruptured dissecting aneurysms of the distal PICA, ${ }^{13}$ parent artery occlusion was carried out in all patients, and 9 patients $(90 \%)$ recovered well with only one death caused by the mass effect of the initial cerebellar hematoma.

The risk of ischemia after PICA occlusion depends on the occlusion site, PICA anatomy, and the presence of collaterals, such as the anterior inferior cerebellar artery, superior cerebellar artery, the contralateral PICA, or a double origin PICA. ${ }^{12,13}$ Occlusion of the PICA is potentially associated with cerebellar infarction distal to the occlusion site, while occlusion at the first 3 proximal segments of the PICA carries potential risk of brain stem ischemia. According to previous reports, collateral flow following distal PICA occlusion may prevent cerebellar ischemia in most cases, and even when limited unilateral cerebellar ischemia occurs it tends to be tolerable. ${ }^{12,13}$ However, attempts should be made to preserve the first 3 proximal segments of the PICA to avoid the potential risk of brain stem ischemia, although the infarction might be limited due to the 
anastomoses of the perforating arteries on the medullary surface. ${ }^{12}$ This was also our experience in the current series. Infarction of the lateral medulla oblongata and Wallenberg's syndrome was observed in one of our patients following occlusion of the aneurysm, as well as the anterior-medullary segment of the PICA.

Despite improvements in endovascular devices and experience, protection techniques using stents or balloons are occasionally limited in treating fusiform or wide-necked aneurysms of the distal PICA. Recently, intravascular stent deployment was safely used in distal small vessels. ${ }^{9}$ However, sometimes the stenosis of the PICA may not even allow catheterization of the vessel, and the small caliber of the PICA may prohibit passage of 2 microcatheters for coiling and stenting simultaneously, as in one of our patients in the present series. In such settings, we tried a modified technique for bailout stent deployment after coiling of the aneurysm. Before detachment of the final coil, the microcatheter for coil delivery was retrieved carefully to the origin of the PICA to make room, navigation of the stent microcatheter and the subsequent stenting was carried out through the inserted 0.014-inch exchange microguidewire. In our experience, this modified technique for bailout stent deployment is an alternative method, if traditional jailing techniques are infeasible due to the insufficient diameter of the PICA for passing 2 microcatheters. In our 2 cases that underwent stent deployment in the PICA, stenting was performed antegrade via the ipsilateral vertebral artery. Retrograde stenting via the contralateral VA has been reported in embolization of wide-necked PICA aneurysms where the origin of that PICA from the VA formed an acute angle. ${ }^{14}$ In a small and tortuous PICA, a microcatheter protective technique is also an alternative to the stent protection for wide-necked aneurysms, which requires positioning an additional microcatheter in the parent artery to protect it. ${ }^{7}$ In this series, we treated one patient using this technique after failure of selective coiling with a single microcatheter. It is easier to navigate a 0.014 microcatheter through a small or acute-angled PICA than a balloon or stent delivery microcatheter. Under the microcatheter protection, the distal portion of the microcatheter covers the neck and prevents coil protrusion.

The recently developed flow-diverting stents, such as the Pipeline Embolization Device (ev3, Irvine, CA, USA) and the SILK flow-diverter (Balt Extrusion, Montmorency, France), provide a new therapeutic choice of intraluminal reconstruction of the diseased vessel in treating complex aneurysms. Although deployment of a flow-diverting stent might be technically challenging in small and tortuous PICA, Alokaili et a ${ }^{15}$ successfully treated a patient with a ruptured distal PICA aneurysm with the placement of a SILK flow-diverter across the aneurysm neck. This therapy may offer a promising treatment option for some distal PICA aneurysms in the future.

In summary, our limited series suggests that distal PICA aneurysms could be managed effectively by various endovascular methods. Protective strategies using a stent or a microcatheter for preserving the proximal PICA segment might be a viable treatment option to reduce the potential risk of brain stem ischemia in select patients. Some limitations of the present study include the small number of cases and the short-term follow-up. Further follow-up and more experience is necessary to determine the long-term results.

\section{References}

1. Bradac GB, Bergui M. Endovascular treatment of the posterior inferior cerebellar artery aneurysms. Neuroradiology 2004; 46: 1006-1011.

2. Lewis SB, Chang DJ, Peace DA, Lafrentz PJ, Day AL. Distal posterior inferior cerebellar artery aneurysms: clinical features and management. J Neurosurg 2002; 97: 756-766.

3. Horiuchi T, Tanaka Y, Hongo K, Nitta J, Kusano Y, Kobayashi S. Characteristics of distal posteroinferior cerebellar artery aneurysms. Neurosurgery 2003; 53: 589-595.

4. Tokimura H, Yamahata H, Kamezawa T, Tajitsu K, Nagayama T, Sugata $S$, et al. Clinical presentation and treatment of distal posterior inferior cerebellar artery aneurysms. Neurosurg Rev 2011; 34: 57-67.

5. Williamson RW, Wilson DA, Abla AA, McDougall CG, Nakaji P, Albuquerque FC, et al. Clinical characteristics and long-term outcomes in patients with ruptured posterior inferior cerebellar artery aneurysms: a comparative analysis. J Neurosurg 2015; 123: 441-445.

6. Lister JR, Rhoton AL Jr, Matsushima T, Peace DA. Microsurgical anatomy of the posterior inferior cerebellar artery. Neurosurgery 1982; 10: $170-199$.

7. Lee JY, Seo JH, Cho YD, Kang HS, Han MH. Endovascular treatment of wide-neck intracranial aneurysms using a microcatheter protective technique: results and outcomes in 75 aneurysms. AJNR Am J Neuroradiol 2011; 32: 917-922.

8. Binning MJ, Hauschild TB, Amini A, MacDonald JD. Delayed posttraumatic saccular aneurysm of PICA in an adolescent. Acta Neurochir (Wien) 2009; 151: 1647-1648.

9. Chen Z, Yang Y, Miao H, Chen J, Luo C, Feng H, et al. Endovascular treatment of ruptured peripheral intracranial aneurysms. Neurosciences (Riyadh) 2012; 17: 133-138.

10. Puri AS, Massari F, Hou SY, Lozano JD, Howk M, Perras M, et al. Onyx embolization in distal dissecting posterior inferior cerebellar artery aneurysms. J Neurointerv Surg 2016; 8: 501-506.

11. Maimon S, Saraf-Lavi E, Rappaport ZH, Bachar G. Endovascular treatment of isolated dissecting aneurysm of the posterior inferior cerebellar artery. AJNR Am J Neuroradiol 2006; 27: 527-732.

12. Isokangas JM, Siniluoto T, Tikkakoski T, Kumpulainen T. Endovascular treatment of peripheral aneurysms of the posterior inferior cerebellar artery. AJNR Am J Neuroradiol 2008; 29: 1783-1788.

13. van den Berg R, Doorschodt TC, Sprengers ME, Vandertop WP. Treatment of dissecting aneurysms of the PICA: anatomical considerations and clinical outcome. J Neuroradiol 2015; 42: 291-297.

14. Cho YD, Kang HS, Lee WJ, Kim KM, Kim JE, Han MH. Stentassisted coil embolization of wide-necked posterior inferior cerebellar artery aneurysms. Neuroradiology 2013; 55: 877-882.

15. Alokaili RN, Ahmed ME. Flow-diverter stent for the treatment of a non-origin posterior inferior cerebellar artery aneurysm. A case report. Neuroradiol J 2014; 27: 456-460. 